Original Paper http://ajol.info/index.php/ijbcs $\quad$ http://indexmedicus.afro.who.int

\title{
Adsorption du benzo(a)pyrène sur du charbon activé à base de coques de coco provenant de Côte d'Ivoire
}

\author{
Urbain Paul GNONSORO ${ }^{1,2 *}$, Koffi Marcellin YAO $^{2}$, Brou Lazare YAO $^{3}$, \\ Aka Marcel KOUASSI ${ }^{2}$, Ardjouma DEMBÉLÉ ${ }^{3}$, Yao Urbain KOUAKOU ${ }^{1}$, \\ Kalédia Paul Honoré OUATTARA ${ }^{1}$, Donourou DIABATÉ ${ }^{1}$ et Albert TROKOUREY ${ }^{1}$ \\ ${ }^{1}$ Laboratoire de Chimie Physique, Université Félix Houphouët Boigny, 22 BP 582 Abidjan 22, Côte d'Ivoire. \\ ${ }^{2}$ Centre de Recherche Océanologique (CRO), BP V18 Abidjan, Côte d'Ivoire. \\ ${ }^{3}$ Laboratoire Central d'Agrochimie et d'Ecotoxicologie (LCAE), 04 BP 612 Abidjan 04, Côte d'Ivoire. \\ *Auteur correspondant ; E-mail: pugues2002@yahoo.fr; Tel (225) 07440725
}

\section{RESUME}

L'accumulation des Hydrocarbures Aromatiques Polycycliques (HAP) dans l'environnement est une préoccupation mondiale du fait de leur persistance et de leur toxicité. l'Union Européenne a ainsi jugé seize HAP comme «préoccupants », parmi lesquels le benzo(a)pyrène est l'un des plus toxiques. La dépollution du benzo(a)pyrène contenu dans les eaux a ainsi été étudiée par adsorption sur charbon activé préparé à partir de coques de noix de coco. La concentration résiduelle en benzo(a)pyrène et l'influence de la masse de charbon activé étudiées par Chromatographie Liquide à Haute Performance (CLHP) ont montré que la quantité de benzo(a)pyrène adsorbée dans l'eau croissait avec la masse de charbon activé introduite. L'adsorption du benzo(a)pyrène a suivi les modèles de Freundlich et de Langmuir et a obéit à la cinétique d'ordre 2; avec une constante thermodynamique $\left(\mathrm{K}_{\mathrm{ad} 2}\right)$ de $0,0706 \mathrm{~g} \cdot \mu \mathrm{g}^{-1} \cdot \mathrm{min}^{-1}$. Aussi, le paramètre d'équilibre $k$ inférieur à 1 et le facteur d'hétérogénéité $n$ compris entre 0,5 et 1 ont indiqué que l'adsorption du benzo(a)pyrène dans l'eau était favorable, cependant le charbon étudié a présenté un pouvoir adsorbant faible pour l'adsorption du benzo(a)pyrène. Ainsi, Le charbon activé à base de coques de coco est donc un moyen limité de dépollution du benzo(a)pyrène.

(c) 2015 International Formulae Group. All rights reserved.

Mots clés: Adsorption, charbon activé, benzo(a)pyrène, isotherme, cinétique.

\section{Benzo(a)pyrene adsorption on activated coal based on coconut shells from Côte d'Ivoire}

\begin{abstract}
Polycyclic Aromatic Hydrocarbons (PAHs) accumulation in environment is a world preoccupation, due to their persistence, toxicity and carcinogenic character for the food chain. The European union judged sixteen thus among them " preoccupying ", among which the benzo(a)pyrene is one of the most toxic. Thus, removal of benzo(a)pyrene from waters has been studied by adsorption on activated carbon prepared from raw coconut cockles. The vestigial concentration in benzo(a)pyrène and the influence of activated carbon mass studied by a High Performance Liquid Chromatography (HPLC), showed that the quantity of benzo(a)pyrene adsorbed in
\end{abstract}


water grows with carbon mass introduced. Benzo(a)pyrene adsorption follows the Freundlich and Langmuir models and it obeys the kinetics of order 2; with a thermodynamic constant $\left(\mathrm{K}_{\mathrm{ad} 2}\right)$ of $0,0706 \mathrm{~g} \cdot \mu \mathrm{g}^{-1} \cdot \mathrm{min}^{-1}$. Also, the balance parameter $k$ lower to 1 and the heterogeneity factor $n$ comprise between 0,5 and 1 indicate that benzo(a)pyrene adsorption in water is favorable, however the studied carbon presents a weak adsorption power for benzo(a)pyrène. So, activated carbon prepared from coconut cockles is therefore a limited method of benzo(a)pyrene removal from waters.

(c) 2015 International Formulae Group. All rights reserved.

Keywords: Adsorption, activated carbon, benzo(a)pyrene, isotherm, kinetics.

\section{INTRODUCTION}

Les Hydrocarbures Aromatiques Polycycliques (HAP) sont des composés organiques hydrophobes issus de la combustion incomplète des matières carbonées. Ces polluants sont des composés ubiquistes détectés dans tous les écosystèmes, des régions polaires aux tropiques (Wilcke, 2007) et du fait de leurs propriétés cancérigène, mutagène et toxique, les HAP sont considérés comme polluants prioritaires (Danyi et al., 2009; Gomez-Ruiz et Wenzl, 2009). L'agence Américaine de Protection Environnementale (USEPA) et l'Union Européenne ont ainsi identifié 16 HAP comme polluants prioritaires du fait de leur grande distribution dans l'environnement et leur risque potentiel pour l'homme (Buco et al., 2004). Au nombre de ces polluants, le benzo(a)pyrène est considéré comme l'un des plus toxiques. La présence du benzo(a)pyrène a été mise en évidence dans plusieurs sphères de l'environnement de plusieurs pays en voie de développement (PED) dont les décharges publiques. En effet, le lixiviat issu de ces décharges peut contenir d'importantes concentrations de polluants chimiques tels que des HAP parmi lesquels le benzo(a)pyrène (Mor et al., 2006 ; Arukwe et al., 2012) qui est une source potentielle de pollution de l'environnement.

Il apparaît donc important, même nécessaire de trouver des moyens pour éliminer cette substance chimique des eaux. Bien qu'il existe plusieurs modes de traitement des eaux, parmi lesquels la coagulation, l'osmose inverse, la précipitation chimique, la filtration sur membrane etc., l'adsorption sur un adsorbant approprié est le moyen le plus prometteur et techniquement efficace (Athéba et al., 2015). Cependant, le coût souvent élevé de certains adsorbants est un frein à l'application d'une telle méthode. L'utilisation de charbons activés préparés à partir de matériaux moins onéreux ou de déchets tels que la coque brute de noix de coco est de plus en plus répandue.

La présente étude vise comme objectifs à proposer un moyen de dépollution du benzo(a)pyrène contenu dans les eaux et à valoriser un moyen de dépollution à partir de déchets agricoles tels que la coque brute de noix de coco.

\section{MATERIEL ET METHODES}

\section{Les solutions aqueuses de benzo(a)pyrène}

Une solution mère de benzo(a)pyrène de $200 \mu \mathrm{g} / \mathrm{l}$ a été préparée dans l'eau distillée à partir d'une solution commerciale de 10.000 $\mu \mathrm{g} / \mathrm{l}$ fournie par Dr Ehrenstorfer $\mathrm{GmbH}$ (Ausburg, Allemagne) et conservée à l'abri de la lumière. Les solutions de différentes concentrations utilisées dans le cadre de cette étude ont été ensuite obtenues par dilution de la solution mère.

\section{Le charbon actif}

Le charbon actif utilisé tout au long de cette étude, a été préparé au Laboratoire d'Agrochimie et d'Ecotoxicologie d'Abidjan, Côte d'Ivoire (LCAE). Les coques brutes de noix de coco ont été d'abord séchées à 105 
${ }^{\circ} \mathrm{C}$, imprégnées de $\mathrm{NaOH}$, puis carbonisées à $500{ }^{\circ} \mathrm{C}$ pendant 5 heures dans un four électrique (Advantec KL-280). Les caractéristiques du charbon actif sont résumées dans le Tableau 1.

\section{Méthode de dosage}

Les concentrations de benzo(a)pyrène absorbé au cours de cette étude ont été déterminées par mesure de la concentration résiduelle du benzo(a)pyrène dans la phase liquide après extraction liquide/liquide, purification et lecture par Chromatographie Liquide à Haute Performance (CLHP) de type SHIMADZU, model LC-20AT muni d'un détecteur UV/Visible à une longueur d'onde de $296 \mathrm{~nm}$.

\section{Description des essais d'adsorption}

Pendant l'étude, les essais d'adsorption ont été réalisés en mettant en contact $10 \mathrm{ml}$ de solution aqueuse de benzo(a)pyrène avec une masse bien déterminée de charbon activé de noix de coco. Chaque échantillon a été agité de façon continue à l'aide d'un agitateur magnétique pendant un temps d'équilibre bien déterminé, puis le mélange a été centrifugé à 2000 tours/min pendant $5 \mathrm{~min}$. Le surnageant a été extrait et dosé par CLHP après extraction et purification (Bowman et al., 2002). La quantité de benzo(a)pyrène adsorbée par le charbon activé est déterminée par la relation suivante:

$Q_{e}=\frac{V_{x}\left(C_{0}-C_{e}\right)}{m}$

Avec: Qe: capacité de rétention du Benzo(a)pyrène par unité de masse de charbon $(\mu \mathrm{g} / \mathrm{g})$;

Ce: concentration de Benzo(a)pyrène à l'équilibre dans la phase aqueuse $(\mu \mathrm{g} / \mathrm{l})$;

$\mathrm{C}_{0}$ : concentration initiale en Benzo(a)Pyrène dans la phase aqueuse $(\mu \mathrm{g} / \mathrm{l})$;
$\mathrm{V}$ : volume de la solution initiale en Benzo(a)Pyrène (1);

$\mathrm{m}$ : masse de charbon $(\mathrm{g})$.

Nous avons commencé les essais par l'étude de la cinétique d'adsorption en faisant agiter des solutions aqueuses de benzo(a)pyrène de concentrations $10 ; 7 ; 5 ; 2$, et $1 \mu \mathrm{g} / \mathrm{l}$ avec une masse de $0,05 \mathrm{~g}$ de charbon activé pendant 1 heure $30 \mathrm{~min}$. Les prélèvements au cours du temps, ainsi que le dosage de la teneur résiduelle $\mathrm{du}$ benzo(a)pyrène ont permi de suivre la cinétique de sa rétention sur le charbon activé utilisé. L'influence de la masse de charbon activé $(0,005 \mathrm{~g}$ à $0,3 \mathrm{~g}$ dans $10 \mathrm{ml}$ de solution à $1 \mu \mathrm{g} / \mathrm{l}$ de benzo(a)pyrène) a également été étudiée. Le $\mathrm{pH}$ durant toute cette étude a été fixé à 8.

\section{RESULTATS \\ Cinétique d'adsorption}

L'étude de l'influence du temps d'agitation sur la cinétique d'adsorption du benzo(a)pyrène a été effectuée pendant 1 heure $30 \mathrm{~min}$ d'agitation. Les résultats obtenus (Figure 1) montrent une diminution de la concentration résiduelle de benzo(a)pyrène avec le temps d'agitation avec apparition d'un palier correspondant à un maximum d'adsorption après 15 minutes, suivie d'une réduction plus lente de la croissance résiduelle jusqu'aux alentours de 30 minutes lors de la deuxième étape. Au-delà de ce temps, la concentration résiduelle du benzo(a)pyrène en solution reste pratiquement stable.

\section{Modélisation de la cinétique d'adsorption}

La modélisation de la cinétique d'adsorption du benzo(a)pyrène a été faite à l'aide des équations cinétiques du premier ordre (modèle Lagergren) et du second ordre. 


\section{Equation du premier ordre}

Lagergren a proposé en 1898 (Srivastava et al., 2006) un modèle cinétique du premier ordre exprimé par la relation suivante :

$$
\frac{d q}{d t}=K_{a d 1}\left(a_{m}-q\right)
$$

Avec $q_{m}$

déterminée

expérimentalement, représentant la quantité maximale adsorbée $(\mu \mathrm{g} / \mathrm{g}), q$ la quantité adsorbée à l'instant $t(\mu \mathrm{g} / \mathrm{g})$ et $k_{\text {adl }}$ la constante du taux d'adsorption du pseudo-premier ordre $\left(\min ^{-1}\right)$. forme :

L'équation 2 peut se mettre sous la

$$
\frac{d q}{q_{n}-q}=K_{a d 1} d t
$$

L'intégration de l'équation 3 donne (Weng et Huang, 2004)

$$
\ln \left(q_{m}-q\right)=\ln q_{m}-K_{a d 1} t
$$

Si ce modèle décrit correctement la cinétique d'adsorption, la représentation graphique de l'équation 4 sera une droite avec $k_{a d l}$ la pente de cette droite et $\ln q_{m}$ l'ordonnée à l'origine. Les paramètres cinétiques de ce modèle pourront être déduits de la représentation graphique de la droite

$$
\ln \left(a_{m}-q\right)=f(t)
$$

\section{Equation du deuxième ordre}

Le modèle du pseudo-deuxième ordre suggère l'existence d'une chimisorption, un échange d'électron par exemple entre molécule d'adsorbât et l'adsorbant solide. Il est représenté par la formule suivante (Kumar, 2007) :

$$
\frac{d q}{d t}=K_{a d 2}\left(a_{m}-q\right)^{2}
$$

$q$ et $q_{m}$ représentent les mêmes grandeurs que dans l'équation $2, k_{a d 2}$ est la constante du taux d'adsorption du pseudo-deuxième ordre (g. $\mu \mathrm{g}^{-}$ $\left.{ }^{1} . \mathrm{min}^{-1}\right)$.

Cette équation peut s'écrire :

$$
\frac{d q}{a_{m}-a^{2}}=F_{a d 2} d t
$$

L'intégration de l'équation 7 donne :

$$
\frac{1}{q_{m}-q}-\frac{1}{q_{m}}=K_{a d 2} t
$$

La forme modifiée de l'équation 8 est utilisée pour les études d'adsorption :

$$
\begin{aligned}
& \frac{\left.a_{m}-a_{m}-a\right)}{\left.a_{m}-q_{m}-a\right)}=K_{a d 2} t \\
& K_{a d 2} t=\frac{q}{q^{2}-a_{m}} \\
& \frac{1}{K_{a d 2} t}=\frac{q^{2}-q \cdot q_{m}}{q} \\
& \frac{1}{K_{a d 2} t}=\frac{q_{m}^{2}}{q}-q_{m} \\
& \frac{a_{m}{ }^{2}}{q}=\frac{1}{K_{a d 2}}+q_{m}
\end{aligned}
$$

La multiplication de tous les termes de l'équation 13 par $\frac{1}{9_{y_{i}}^{2}}$ donne (Horsfallet Spiff, 2004):

$$
\frac{t}{q}=\frac{1}{K_{a d 2} q_{m}^{2}}+\frac{t}{q_{n}}
$$

$$
\text { La représentation } \mathrm{t} / \mathrm{q}=\mathrm{f}(\mathrm{t}) \text {, où } \frac{1}{q_{m}} \text { est la }
$$
pente et $\frac{1}{K_{\Delta \sigma_{2}} a_{m_{2}}{ }^{2}}$ est l'ordonnée à l'origine, permet de déterminer les paramètres cinétiques de ce modèle, notamment la constante cinétique $k_{a d 2}$ et la capacité d'adsorption à l'équilibre $q_{m}$. Cette représentation serait une droite si ce modèle décrit bien la réaction d'adsorption.

Les paramètres associés aux modèles du premier et du second ordre sont consignés 
dans le Tableau 2. Le coefficient de corrélation de la réaction de pseudo-ordre 2 $(0,999)$ est très proche de 1 comparativement à la réaction de pseudo-ordre 1 et la quantité maximale adsorbée $\left(q_{m}\right)$ calculée dans l'équation de pseudo-second ordre est plus proche de la valeur expérimentale. En effet, les quantités maximales adsorbées $\left(q_{m}\right)$ calculées par les équations de pseudo-ordre 1 et 2 sont respectivement de 49,353 $\mu \mathrm{g} / \mathrm{g}$ et de $31,153 \mu \mathrm{g} / \mathrm{g}$ sachant que $q_{m}$ expérimentale est de $30,876 \mu \mathrm{g} / \mathrm{g}$.

\section{Isothermes d'adsorption du benzo(a)pyrène}

Les isothermes d'adsorption jouent un rôle important dans la détermination des capacités maximales d'adsorption, et dans la conception de nouveaux adsorbants. Il est donc indispensable de les déterminer (Athéba et al., 2015).

\section{Isotherme de Freundlich}

La relation empirique de l'isotherme de Freundlich est de la forme (Al Mardini, 2008) $\mathrm{Q}_{\mathrm{e}}=\mathrm{K}_{\mathrm{f}} \mathrm{C}_{\mathrm{e}}^{1 / \mathrm{n}}$

Avec :

$\mathrm{Q}_{\mathrm{e}}$ : Capacité d'adsorption en $\mu \mathrm{g}$ de soluté adsorbé par g d'adsorbant,

$\mathrm{C}_{\mathrm{e}}$ : Concentration à l'équilibre du soluté en phase liquide $(\mu \mathrm{g} / \mathrm{L})$,

$1 / \mathrm{n}$ et $\mathrm{K}_{\mathrm{f}}$ : Coefficient et constante d'adsorption respectivement.

La constante de Freundlich $\left(\mathrm{K}_{\mathrm{f}}\right)$ traduit le pouvoir adsorbant d'une matrice vis-à-vis de l'adsorbant considéré. Plus la valeur de $\mathrm{K}_{\mathrm{f}}$ est élevée, plus l'adsorption est importante. La transformée linéaire permettant de vérifier la validité de l'équation (15) est obtenue par passage en échelle logarithmique:

$$
\eta Q_{e}=m K_{f}+\frac{1}{n} m C_{\theta}
$$

Si la représentation graphique de $\ln Q_{e}$ en fonction de $\ln C_{e}$ donne une droite, on peut conclure que l'équation de Freundlich est applicable. Ce qui permet de calculer les constantes $k$ et $n$ (Carvalho et al., 2006). L'application du modèle de Freundlich à l'adsorption du benzo(a)pyrène sur le charbon activé de noix de coco a donné un coefficient de corrélation de 0,847 , une constante de Freundlich $k$ de 0,1083 et un facteur d'hétérogénéité $n$ compris entre 0,5 et 1 (Tableau 3). Sachant que, plus la constante de Freundlich $k$ est élevée, meilleure est l'adsorption (Ouattara, 2014), l'adsorption du benzo(a)pyrène sur le charbon activé de coque brute de noix de coco (CAC) est bonne, car la constante de Freundlich $k$ est de 0,1083.

\section{Isotherme de Langmuir}

Le modèle de Langmuir permet de calculer la capacité d'adsorption maximale des matériaux adsorbants (Avom et al., 2001). Le modèle de Langmuir est représenté mathématiquement par l'équation suivante :

$$
q_{e}=\frac{q_{m} b c_{e}}{1+b c_{e}}
$$

La linéarisation de cette équation (17) permet d'écrire :

$$
\frac{1}{q_{e}}=\frac{1}{q_{m}}+\frac{1}{q_{m} b} \times \frac{1}{c_{e}}
$$

Avec $\boldsymbol{b}$ la constante thermodynamique de Langmuir liée à l'énergie libre d'adsorption,

$\boldsymbol{F}$ ( $(\mu \mathrm{g} / \mathrm{g})$ la quantité de soluté adsorbée par unité de masse d'adsorbant à l'équilibre et $q_{m}(\mu \mathrm{g} / \mathrm{g})$ la quantité de soluté adsorbée par gramme de solide nécessaire pour recouvrir la surface de l'adsorbant d'une couche monomoléculaire ou la capacité maximum d'adsorption. $\quad C_{\bullet}(\mu \mathrm{g} / \mathrm{L})$ représente la concentration résiduelle du soluté à l'équilibre (Hamdaoui et Naffrechoux, 2007). 
La pente de la représentation graphique de $\frac{1}{q_{e}}$ en fonction de $\frac{1}{c_{e}}$ permet de calculer les constantes $q_{m}$ et $b$.

Le coefficient de corrélation de l'isotherme de Langmuir de 0,999 et la valeur positive $\mathrm{du}$ coefficient $\mathrm{b}$ (Tableau 3), traduisent que l'adsorption du benzo(a)pyrène sur le charbon activé de noix de coco est spontanée et obéit au modèle de Langmuir.

\section{Effet de la masse du charbon actif}

L'influence de la masse du charbon activé de noix de coco sur la capacité d'adsorption du benzo(a)pyrène a été étudiée et a consisté à faire varier la quantité du charbon actif tout en conservant la concentration initiale du benzo(a)pyrène en solution. En effet, la masse de charbon actif a varié de $0,005 \mathrm{~g}$ à $0,3 \mathrm{~g}$ pour une teneur initiale constante en benzo(a)pyrène de $1 \mu \mathrm{g} / \mathrm{L}$ dans $0,01 \mathrm{~L}$ de solution et une agitation de 30 minutes. Les résultats obtenus, présentés à la Figure 2 montrent que la quantité de benzo(a)pyrène adsorbée croît avec la masse de charbon activé de noix de coco introduite.

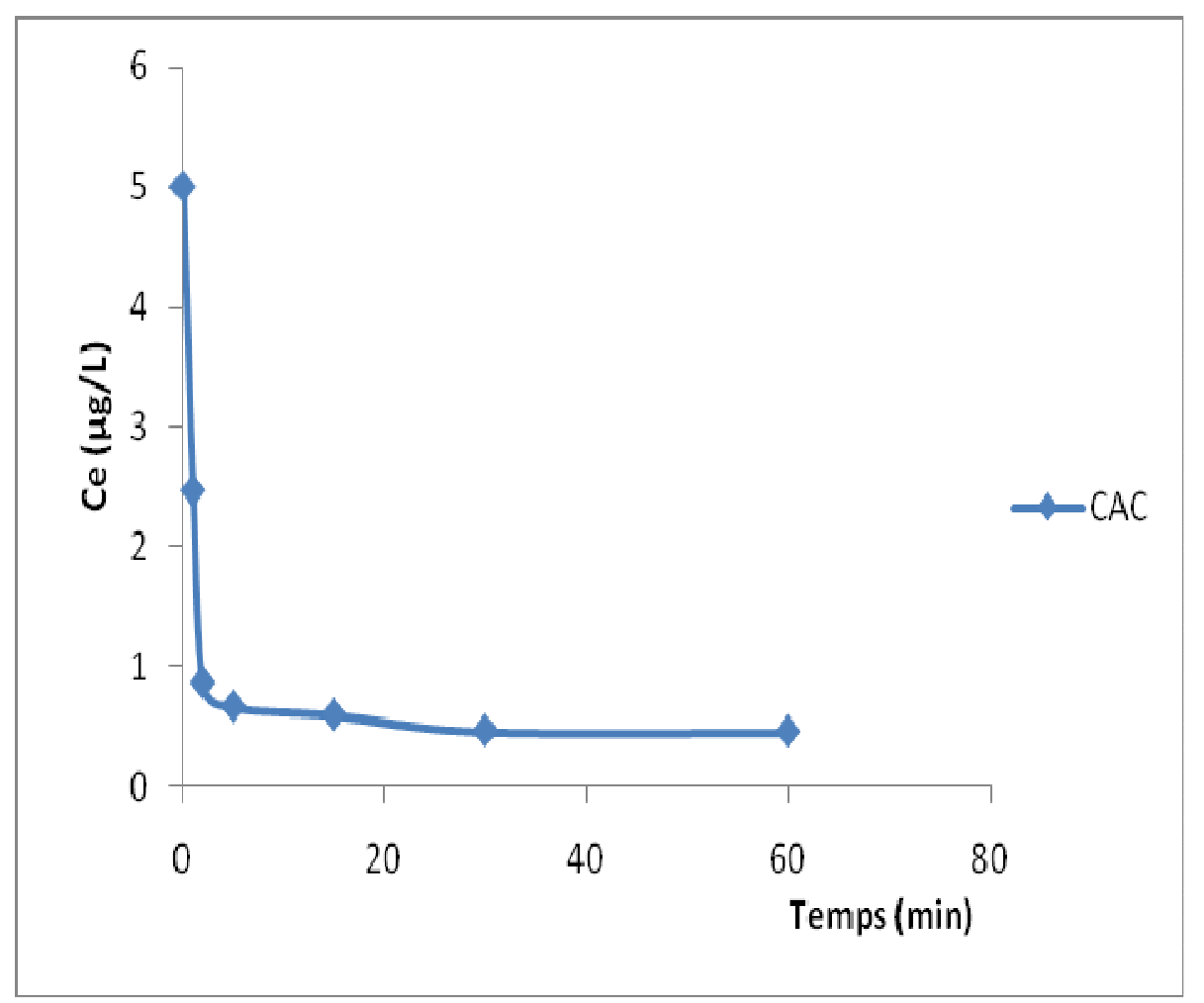

Figure 1 : Cinétique d'adsorption du benzo(a)pyrène sur le charbon activé de noix de coco. Ce: concentration de Benzo(a)pyrène à l'équilibre dans la phase aqueuse. 


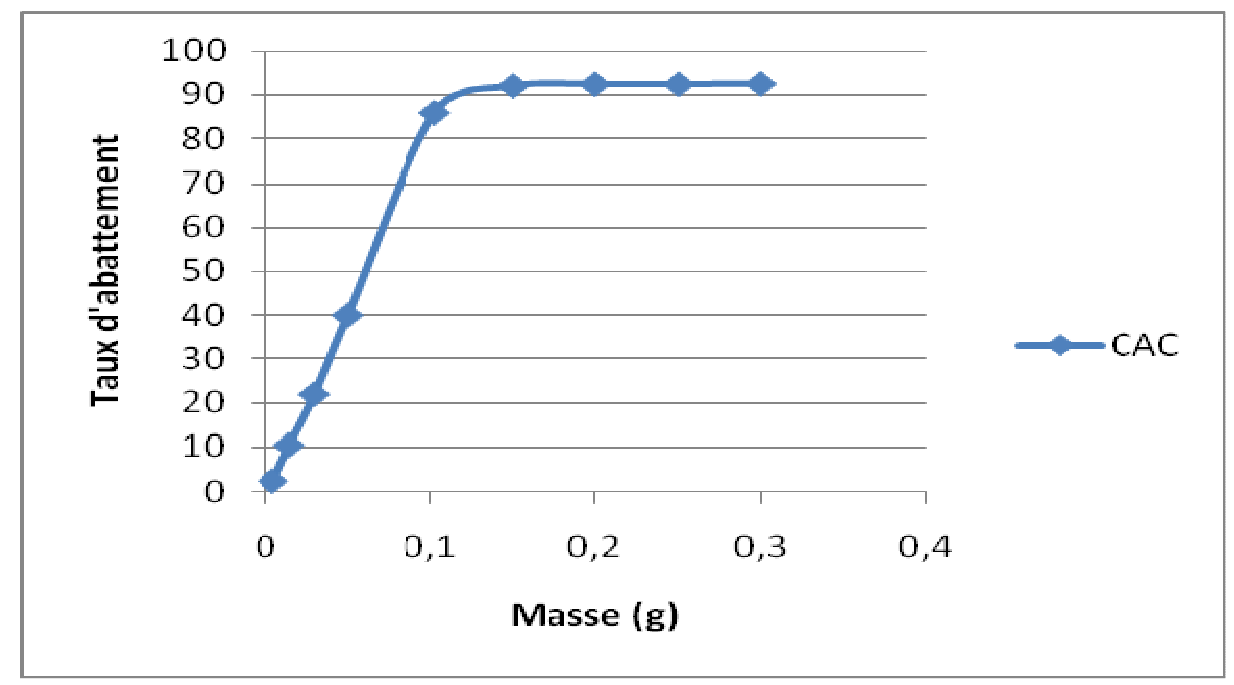

Figure 2 : Influence de la masse d'adsorbant sur l'adsorption du benzo(a)pyrène.

Tableau 1: Caractéristiques du charbon actif de la coque de coco.

\begin{tabular}{|c|c|c|c|}
\hline \multicolumn{4}{|c|}{ Charbon activé de coque de coco (CAC) } \\
\hline \multirow{3}{*}{ Physiques } & \multicolumn{2}{|c|}{$\mathrm{Qm}(\mathrm{mmol} / \mathrm{g})$} & 5,74 \\
\hline & \multicolumn{2}{|c|}{$\mathrm{R}^{2}$} & 0,994 \\
\hline & \multicolumn{2}{|c|}{$\mathrm{S}_{\mathrm{BET}}\left(\mathrm{m}^{2} / \mathrm{g}\right)$} & 725 \\
\hline \multirow{6}{*}{$\begin{array}{l}\text { Chimiques } \\
\text { (Boehm, pH) }\end{array}$} & \multirow{4}{*}{$\begin{array}{l}\text { Acides } \\
(\mathrm{mmol} / \mathrm{g})\end{array}$} & Carboxylique & 0,29 \\
\hline & & Lactone & 0,21 \\
\hline & & Phénolique & 0,64 \\
\hline & & Surface acide totale & 1,14 \\
\hline & \multicolumn{2}{|c|}{ Surface basique totale } & 1,65 \\
\hline & \multicolumn{2}{|c|}{$\mathrm{pH}$ de surface } & 7,37 \\
\hline \multirow{2}{*}{ Structure } & & ndres $(\%)$ & 4,56 \\
\hline & \multicolumn{2}{|c|}{ Aspect } & Granulé \\
\hline
\end{tabular}

$\mathbf{Q}_{\mathbf{m}}$ : Capacité d'adsorption maximale en $\mu \mathrm{g}$ de soluté adsorbé par g d'adsorbant

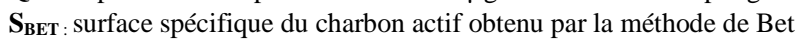
pH : potentiel d'hydrogène

Tableau 2: Paramètres caractérisant la cinétique d'adsorption du benzo(a)pyrène sur charbon actif.

\begin{tabular}{|c|c|c|c|c|c|c|c|c|}
\hline \multirow{2}{*}{$\begin{array}{c}q_{m} \text { expérimentale } \\
(\mu \mathrm{g} / \mathrm{g})\end{array}$} & \multicolumn{4}{|c|}{ Cinétique d'ordre 1} & \multicolumn{4}{|c|}{ Cinétique d'ordre 2} \\
\hline & $\begin{array}{c}q_{m} \\
\text { calculée } \\
(\mu \mathrm{g} / \mathrm{g})\end{array}$ & $\begin{array}{c}k_{a d 1} \\
\left(\min ^{-1}\right)\end{array}$ & $\mathbf{R}^{2}$ & $\begin{array}{l}\Delta q m \\
(\%)\end{array}$ & $\begin{array}{c}q_{m} \text { calculée } \\
(\mu \mathrm{g} / \mathrm{g})\end{array}$ & $\begin{array}{c}k_{\text {ad } 2} \\
\left(\mathrm{~g} \cdot \mu \mathrm{g}^{-1} \cdot \min ^{-1}\right)\end{array}$ & $\mathbf{R}^{2}$ & $\begin{array}{l}\Delta q m \\
(\%)\end{array}$ \\
\hline 30,876 & 49,353 & 0,317 & 0,940 & 59,84 & 31,153 & 0,0706 & 0,999 & 0,90 \\
\hline
\end{tabular}

$\boldsymbol{q}_{\boldsymbol{m}}$ : Capacité d'adsorption maximale en $\mu \mathrm{g}$ de soluté adsorbé par g d'adsorbant ; $\boldsymbol{k}_{\text {adl }}$ : constante du taux d'adsorption de la réaction de pseudo-premier ordre ; $\boldsymbol{k}_{a d 2}$ : constante du taux d'adsorption de la réaction de pseudo-deuxième ordre ; $\Delta \boldsymbol{q} \boldsymbol{m}:$ taux d'écart entre $q_{m}$ expérimentale et $q_{m}$ calculée . 
Tableau 3 : Paramètres des équations de Langmuir et de Freundlich.

\begin{tabular}{cccccc}
\hline & Freundlich & \multicolumn{3}{c}{ Langmuir } \\
\hline \multirow{2}{*}{$\mathbf{R}^{2}$} & $\boldsymbol{k}$ & $\boldsymbol{n}$ & $\mathbf{R}^{2}$ & $\boldsymbol{q}_{\boldsymbol{m}}(\boldsymbol{\mu g} / \mathbf{g})$ & $\boldsymbol{b}$ \\
& {$\left[(\boldsymbol{\mu g} / \mathbf{g})(\mathbf{L} / \mu \mathbf{g})^{\frac{1}{n}}\right]$} & & & & \\
\hline 0,847 & 0,1083 & 0,96 & 0,999 & 3,34 & 0,0179 \\
\hline
\end{tabular}

\section{DISCUSSION}

\section{Cinétique d'adsorption}

L'adsorption du benzo(a)pyrène sur le charbon activé de noix de coco est rapide et peut-être scindée en deux étapes. La première étape consiste en une croissance rapide de l'adsorption pendant les 15 premières minutes, suivie d'une réduction plus lente de la croissance résiduelle jusqu'aux alentours de 30 minutes lors de la deuxième étape. Au-delà de ce temps, la concentration résiduelle du benzo(a)pyrène en solution reste pratiquement stable. Ce résultat ne fait que confirmer l'influence de la surface spécifique sur l'adsorption, puisque la stabilité observée s'explique par l'occupation des sites d'adsorption du charbon actif par les molécules de l'adsorbât (Athéba et al., 2015). Des résultats similaires ont été observés par Anouar et al. (2014) pour l'adsorption de l'orange 16 colorant azoïque sur charbon actif en poudre.

\section{Modélisation de la cinétique d'adsorption}

Le coefficient de corrélation de la réaction de pseudo-ordre $2(0,999)$ plus proche de 1 que la réaction de pseudo-ordre 1 $(0,940)$ et la quantité maximale adsorbée $\left(q_{m}\right)$ calculée dans l'équation de pseudo-second ordre plus proche de la valeur expérimentale, prouve que l'adsorption du benzo(a)pyrène sur le charbon actif étudié obéit à la cinétique de pseudo-ordre 2. Chen et al. (2010) ont déterminé aussi une réaction d'ordre 2 lors de l'étude de l'adsorption de l'oxyde de méthyle et de butyle sur le charbon activé en grain.

\section{Isothermes d'adsorption du benzo(a)pyrène}

L'étude des isothermes d'adsorption du benzo(a)pyrène a montré que le modèle de Langmuir s'applique mieux que celui de Freundlich. En effet, le coefficient de corrélation lié à l'adsorption du benzo(a)pyrène suivant le modèle de Langmuir $(0,999)$ est plus proche de 1 que celui de Freundlich $(0,847)$ (Tableau3). Ainsi, l'adsorption du benzo(a)pyrène sur le charbon activé de noix de coco est spontanée et obéit au modèle de Langmuir. Aussi, l'application du modèle de Langmuir a permis de calculer la capacité d'adsorption maximale $9 \mathrm{~m}$ nécessaire pour recouvrir la surface du charbon actif. La valeur de $q_{m}=3,34 \mu \mathrm{g} / \mathrm{g}$ indique que la quantité adsorbée à l'équilibre est assez faible. Cette faible capacité d'adsorption est due au fait qu'en présence d'une concentration faible en adsorbât, les forces de diffusion du soluté vers l'adsorbât deviennent plus faibles (Athéba et al., 2015). Aussi, le facteur d'hérogénéité $n$ du model de Freundlich compris entre 0,5 et 1 , est la preuve d'une affinité moyenne, mais assez bonne entre l'adsorbant et l'adsorbât. Cela démontre aussi que l'adsorption observée est de nature physique.

\section{Effet de la masse du charbon actif}

La croissance de la quantité de benzo(a)pyrène adsorbée avec la masse de charbon activé introduite est due à l'accroissement des sites d'adsorption à la surface du charbon actif (Athéba et al., 2015). 
Des observations similaires ont été faites par Bouchemal et al. (2007) lors de l'étude de l'adsorption de la tyrosine sur charbon actif en grains et en poudre. Le maximum d'élimination ou d'abattement du benzo(a)pyrène $(92 \%)$ a été atteint pour une masse de $0,15 \mathrm{~g}$ de charbon activé de noix de coco.

\section{Conclusion}

L'étude expérimentale que nous avons menée a été consacrée à l'évaluation de la capacité d'adsorption du benzo(a)pyrène sur un charbon activé à base de coques de noix de coco d'origine ivoirienne afin de contribuer à son élimination des ressources aquatiques. L'effet du temps et de la masse de charbon ont été pris en compte et ont conduit à plusieurs résultats. L'équilibre d'adsorption a été atteint au bout de 30 minutes d'agitation et l'adsorption du benzo(a)pyrène a augmenté avec la masse du charbon activé de noix de coco introduite. Le maximum d'élimination ou d'abattement du benzo(a)pyrène (92\%) en solution aqueuse de concentration $1 \mu \mathrm{g} / \mathrm{L}$ a été atteint pour une masse de $0,15 \mathrm{~g}$ de charbon activé de noix de coco dans 0,01 L de solution. Aussi, le modèle de Langmuir s'est mieux appliqué que celui de Freundlich pour décrire les isothermes d'adsorption, tandis que la modélisation de la cinétique d'adsorption $\mathrm{du}$ benzo(a)pyrène est d'ordre 2 avec une constante thermodynamique de 0,0706 g. $\mu \mathrm{g}^{-}$ ${ }^{1} \cdot \mathrm{min}^{-1}$.

Les résultats obtenus dans ce travail ont ainsi pu montrer, sur le plan scientifique, que le charbon activé préparé à base de coque de noix de coco présente un pouvoir adsorbant faible pour l'adsorption du benzo(a)pyrène.

\section{Conflit d'intérêts}

Les auteurs déclarent n'avoir aucun conflit d'intérêts.

\section{CONTRIBUTIONS DES AUTEURS}

La contribution des auteurs s'est située à tous les niveaux, de la conduite à la réalisation des travaux au laboratoire jusqu'à la rédaction et aux différentes corrections du manuscrit.

En effet, AT et DD ont été les instigateurs du dit projet de recherche et ont aussi été les artisans de sa mise en œuvre en créant le cadre de sa réalisation. AMK a contribué à la mise en œuvre de ce projet, par la création de l'espace de travail et par la fourniture d'une partie du matériel. AD et BLY ont aidé à la réalisation du travail en fournissant la paillasse, les produits chimiques, les appareils et les orientations nécessaires ; KMY a de part ses conseils et la coordination des travaux de laboratoire contribuer à l'aboutissement de ce travail, Il a aussi été le principal instigateur de ce manuscrit ; KPHO et YUK ont contribué à la rédaction de ce manuscrit; UPG a été l'investigateur principal de ce projet et l'un des principaux rédacteurs de ce manuscrit.

\section{REMERCIEMENTS}

Les auteurs remercient sincèrement le personnel $\mathrm{du}$ Laboratoire Central d'Agrochimie et d'Ecotoxicologie d'Abidjan (LCAE) pour leur assistance pendant les manipulations.

\section{RÉFÉRENCES}

Al Mardini F. 2008. Etude de l'adsorption du pesticide Bromacil sur charbon actif en poudre en milieu aqueux Effet compétiteur des matières organiques naturelles. Thèse de doctorat, Université de Poitier, France, p. 27.

Anouar H, Elhourch A, Elkacemi K, Zouahri A. 2014. Elimination de l'orange 16 colorant azoique par adsorption sur charbon actif en poudre. Inter. J. Innov. 
$\begin{array}{llll}\text { App. } & \text { St., } & \text { 9(3): } & \text { 1373-1377. }\end{array}$ http://www.ijias.issr-journals.org/

Arukwe A, Eggen T, Möder M. 2012. Solid waste deposits as a significant source of contaminants of emerging concern to the aquatic and terrestrial environments - A developing country case study from Owerri, Nigeria. Sci. Tot. Env., 438(1): 94-102. Doi: 10.1016/j. scitotenv.2012.08.039

Atheba GP, Allou NB, Dongui BK, Kra DO, Gbassi KG, Trokourey A. 2015. Adsorption du butylparabène sur du charbon activé à base des coques de coco provenant de cote d'ivoire. Int. J. Innov. Sci. Res., 13(2): 530-541. http://www.ijisr.issr-journals.org/doi: 10.1016/j.biortech

Avom J, Mbadcam JK, Matip MRL, Germain P. 2001. Adsorption isotherme de l'acide acétique par des charbons d'origine végétale. AJST, 2(2): 1-7.

Bouchemal F, Achour S. 2007. Essais d'adsorption de la tyrosine sur charbon actif en grains et en poudre. Larhyss J., 06: 81-89. Doi: 10.1007/s13201-0140233-3

Bowman JC, Zhou JL, Readman JW. 2002. Sorption and desorption of benzo(a)pyrène in aquatic systems. $J$. Environ. Monit. 4: 761 - 766. Doi: 10.1039/B204969B

Buco S, Moragues M, Doumenq P, Noor A, Mille G. 2004. Analysis of polycyclic aromatic hydrocarbons in contaminated soil by Curie point pyrolysis coupled to gas chromatography-mass spectrometry, an alternative to conventional methods. J. Chrom., 1026: 223-229. Doi: 101016/j.chroma.2003.11.065

Carvalho MF, Duque AF, Goncalves IC, Castro PML. 2006. Adsorption of fluorobenzene onto granular activated carbón: isotherm and bioavailability studies. Bioresource Technology, 98: 3423-3430. doi: 10.1016/ j. biortech. 2006.11.001.

Chen DZ, Zhang JX, Chen JM. 2010. Adsorption of methyl tert-butyl ether using granular activated carbon: Equilibrium and kinetic analysis. Int. J. Environ. Sci. Tech., 7(2): 235-242. http : $\quad$ www.bioline.org.br/pdf\% 3Fst10023

Danyi S, Brose F, Brasseur C, Schneider YJ, Larondelle Y, Pussemier L, Robbens J, De Saeger S, Maghuin-Rogister G, Scippo ML. 2009. Analysis of EU priority polycyclic aromatic hydrocarbons in food supplements using high performance liquid chromatography coupled to anultraviolet, diode array or fluorescence detector. Analyt. Chim. Acta, $\quad$ 663(2): 293-299. Doi:10.1016/J.aca.2008.11.049

Gomez-Ruiz JA, Wenzl T. 2009. Evaluation of gas chromatography columns for the analysis of the $15+1$ EU-priority polycyclic aromatic hydrocarbons (PAHs), Analyt. Bioanalyt. Chem., 393(6-7): $\quad$ 1697-1707. Doi: 10.1007/s00216-008-2585-8.

Hamdaoui O, Naffrechoux E. 2007. Modeling of adsorption isotherms of phenol and chlorophenols onto granular activated carbon. Part I. Two-parameter models and equations allowing determination of thermodynamic parameter. Journal of Hazardous Materials, 147 (1-2), 381-394. Doi: 10.1016/i.jhazmat. 2007.01.021.

Hashimoto AH, Amanuma K, Hiyoshi K, Takano H, Masumura K, Nohmi T, Aoki Y. 2005. In vivo mutagenesis induced by benzo[a]pyrene instilled into the lung of gpt delta transgenic 
mice. Environ. Mol. Mutag., 45(4): 365-373. Doi: 10.1002/em.20098.

Horsfall M, Spiff AI. 2004. Studies on the effect of $\mathrm{pH}$ on the sorption of $\mathrm{Pb}^{2+}$ and $\mathrm{Cd}^{2+}$ ions from aqueous solutions by caladium bicolor (wild cocoyam) biomass. Electron. J. Biotech., 7(3): 17. Doi: $10.2225 / \mathrm{Vol} 7$-issues 3-fulltext8

Kumar KV. 2007. Pseudo-second order models for the adsorption of safranin onto activated carbon: Comparison of linear and non-linear regression methods, Haz. Mat., 142: 564-567. Doi: 10.1016/j.jhazmat.2006.08.018

Mor S, Ravindra K, Dahiya RP, Chandra A. 2006. Leachate Characterization and assessment of groundwater pollution near municipal solid waste landfill site. Environ. Monit. Assess., 4: 325-334. Doi : 10.12691/ajcea-1-2-3.
Ouattara KPH. 2014. Etude de l'adsorption de l'aspirine sur des charbons activés préparés à partir de coques d'arachide (Arachis hypogaea) et de soja vert (vigna radiata), thèse de doctorat, Université Félix Houphouët Boigny d'Abidjan, Côte d'Ivoire, 131p, $\mathrm{N}^{\circ}$ d'ordre 916/2014.

Srivastava VC, Swamy MM, Malli D, Prasad B, Mishra IM. 2006. Adsorptive removal of phenol by bagasse fly ash and activated carbon: Equilibrium, and thermodynamics, Colloids and Surfaces A: Physicoch.and Eng. Asp., 272: 89104. Doi: 10.1016/j.colsurfa. 2005.07.016

Weng CH, Huang CP. 2004. Adsorption characteristics of $\mathrm{Zn}(\mathrm{II})$ from dilute aqueous solution by fly ash. Colloid. Surf. A., 247(1-3): 137-143. Doi: 10.1016/ j.colsurfa.2004.08.050

Wilcke W. 2007. Global patterns of polycyclic aromatic hydrocarbons (PAHs) in soil. Geoderma, 141(3-4): 157-166. 